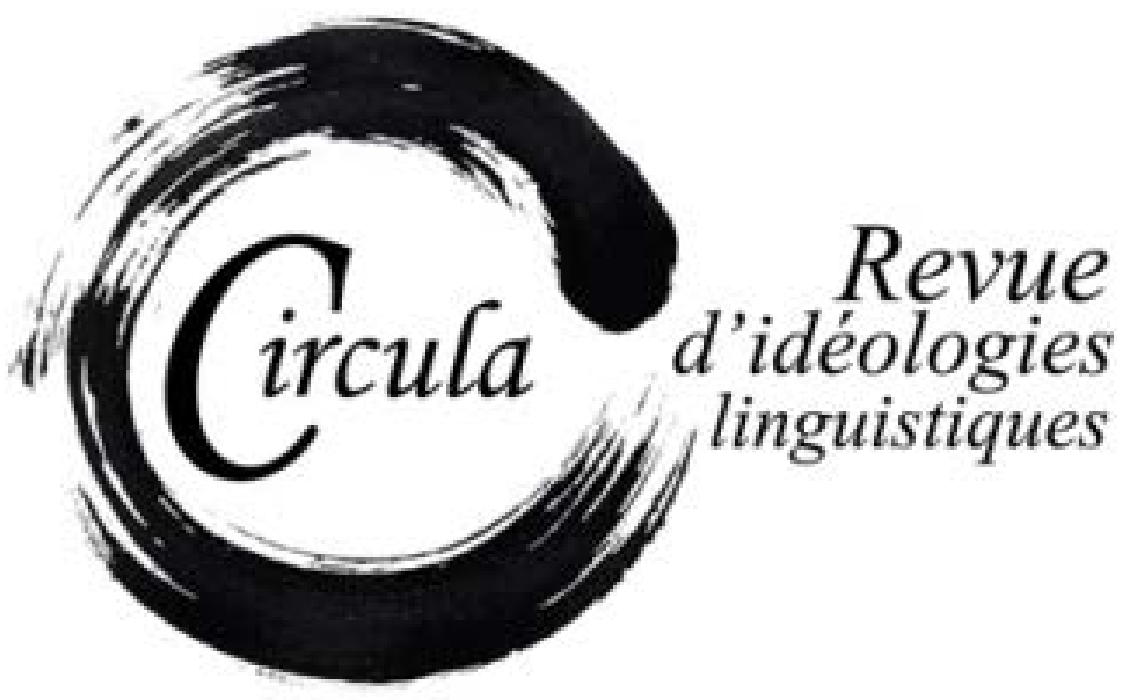

TITRE: UNE LANGUE SANS FUTUR, UNE IDENTITÉ BIEN VIVANTE : REPRÉSENTATIONS DE LA COMMUNAUTÉ ARBËNISHTË

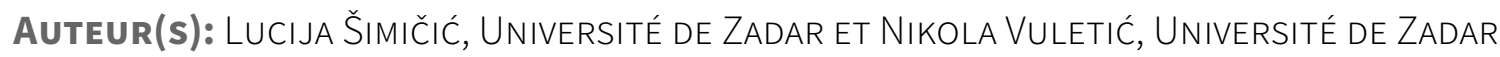

Revue: CirCula, NUMÉRO 3

PAGES: $141-162$

ISSN: 2369-6761

Directeur: Bruno Maurer, Université PAul-VAlÉRy - MontPellier 3

URI: HTTP://HDL.HANDLE.NET/11143/9702

DOI: $10.17118 / 11143 / 9702$ 


\title{
Une langue sans futur, une identité bien vivante : représentations de la communauté arbënishtë
}

\author{
Lucija Šimičić, Université de Zadar \\ lucija.simicic@gmail.com
}

Nikola Vuletić, Université de Zadar

nvuletic@unizd.hr

Résumé : L'arbënishtë est une variété d'albanais traditionnellement parlée dans le quartier d'Arbanasi de la ville de Zadar (Croatie), exposée à la rupture de la transmission générationnelle et à un déclin rapide. Dans cette contribution, nous abordons l'étude de la communauté arbënishtë du point de vue des représentations sociales par les locuteurs de l'arbënishtë, par les adolescents non-locuteurs se disant Arbënishtës et par les non-locuteurs étudiants de l'Université de Zadar. Les différences entre les trois groupes renvoient à une construction discursive de l'identité arbënishtë, à la perception du statut de la langue, ainsi qu'à une relation changeante entre les deux.

Mots-clés : arbënishtë; représentations sociales ; attitudes linguistiques ; substitution linguistique ; ville de Zadar

Summary: Arbënishtë is an Albanian variety traditionally spoken in the quarter of Arbanasi of the city of Zadar (Croatia), exposed to the interruption of the intergenerational transmission and a rapid decline. In this paper, we approach the study of the Arbënishtë community from the point of view of the social representation, as identified by the Arbënishtë speakers, the teenagers who identify as Arbënishtës but do not speak the language, and by a group of non-speakers students of the University of Zadar. The differences found in the three groups point to the discursive construction of the Arbënishtë identity, the perception of the status of the language as well as the changing relation between the two.

Keywords: Arbënishtë; social representations; language attitude; language shift; city of Zadar 


\section{Introduction}

Dans cette contribution, nous proposons une première lecture des résultats de nos enquêtes sur les représentations sociales de la langue arbënishtë, une variété albanaise propre au quartier d'Arbanasi de la ville adriatique de Zadar, en Croatie ${ }^{1}$. Notre recherche a été réalisée dans le cadre du projet "Représentations des langues et des identités en Méditerranée en contexte plurilingue », dirigé par Bruno Maurer de l'Université de Montpellier 3. Les enquêtes, menées selon la méthode d'analyse combinée, un outil d'enquête sociolinguistique développé par Maurer lui-même, concernent trois groupes de représentations sociales de l'arbënishtë : celles propres à cette communauté (autoreprésentations), celles des non-locuteurs (hétéroreprésentations) et celles des adolescents membres de la communauté arbënishtë qui ne parlent pas la langue ancestrale.

Notre contribution se situe dans le cadre de la théorie de la représentation sociale, d'où la nécessité d'offrir d'abord quelques réflexions sur la notion même de représentations sociales, ainsi que sur son rapport avec les attitudes linguistiques, l'un des concepts-clés de la psychologie sociale du langage, notamment de l'étude des langues minoritaires, exposées à la substitution et à la disparition. Étant donné que le contexte social local exerce une influence décisive sur les représentations sociales, ainsi que sur les attitudes linguistiques, nous offrirons, par la suite, une brève description des déterminants historiques et sociolinguistiques de la communauté linguistique arbënishtë. Suite à une analyse descriptive de l'adhésion aux éléments de la représentation (cognèmes; items des questionnaires) et du consensus social sur ceux-ci, accompagnée d'une comparaison des représentations dans les trois groupes qui constituent notre échantillon, nos remarques conclusives portent sur la signification des représentations sociales pour l'étude de la langue et de la communauté arbënishtës. Cette contribution nous servira aussi du point de départ pour une discussion sur les avantages et les inconvénients du cadre théorique utilisé ici dans l'étude des communautés linguistiques en danger.

\section{Représentations sociales et attitudes linguistiques dans l'étude de la substitution linguistique}

Il a été démontré que, parmi les facteurs causant d'une manière directe ou indirecte la substitution linguistique, ceux d'ordre sociopsychologique jouent un rôle prééminent (p. ex. Dorian, 1981 ; Sasse, 1992 ; Bradley, 2002), et qu'ils constituent un aspect indispensable de l'étude de la vitalité ethnolinguistique (Bourhis et Sachdev, 1984 ; Giles, Rosenthal et Young, 1985). Dans la bibliographie sociolinguistique, les attitudes linguistiques et les processus d'identification comptent parmi les facteurs les plus étudiés. Les attitudes linguistiques sont inextricablement liées à des processus plus vastes d'ordre politique, social et/ou économique (Sasse, 1992 ; Tsunoda, 2006). Elles appartiennent

1. Il ne faut pas entendre cette localisation dans les termes absolus : les locuteurs d'arbënishtë sont présents également dans d'autres quartiers de la ville. Néanmoins, le quartier d'Arbanasi constitue le noyau historique de la communauté linguistique arbënishtë. 
à ce que Boyer nomme « imaginaires sociolinguistiques » (Boyer, 1996 : 16). Tandis que les attitudes positives ne garantissent pas forcément le maintien de la langue ni la vitalité ethnolinguistique, les attitudes négatives à l'égard d'une langue donnée ne tarderont pas à provoquer la rupture de la transmission générationnelle de cette langue, son déclin et, à long terme, sa perte (Sasse, 1992).

Il est possible d'adresser deux critiques principales au choix des attitudes comme base méthodologique dans l'étude du maintien et de la substitution linguistiques. D'abord, il faut tenir compte du caractère individuel du concept d'attitude (p. ex. Doise, 1989), qui se révèle problématique dans l'étude du langage comme une construction éminemment sociale. Un autre problème concernant la fiabilité de l'étude des attitudes dans une recherche (linguistique) quelconque, c'est la complexité inhérente du concept même, ainsi que son fonctionnement à plusieurs niveaux (Kristiansen et Jørgenssen, 2005), ce qui rend impossible la prédiction du comportement (l'usage effectif de la langue, en l'occurrence) à partir des attitudes linguistiques, telles qu'elles sont exprimées par les locuteurs eux-mêmes². Face à ces inconvénients, il est utile de faire recours à la théorie de la représentation sociale.

Promu par Moscovici (1961, 1963, 1988, etc.) comme «système de valeurs, idées et pratiques » (Moscovici, 1973 : xiii), le concept de représentation sociale est aujourd'hui généralement entendu comme « l'ensemble des croyances, des connaissances et des opinions qui sont produites et partagées par les individus d'un même groupe, à l'égard d'un objet social donné » (Guimelli, 1999 : 63)³. Selon Abric, la représentation sociale est « déterminée à la fois par le sujet lui-même (son histoire, son expérience), par le système social et idéologique dans lequel il est inséré, et par la nature des liens que le sujet entretient avec le système social » (Abric, 1989 : 188). On attribue aux représentations sociales quatre groupes de fonctions - cognitives, identitaires, d'orientations, et justificatives (Abric 1994 : 16-17) - permettant à l'individu de comprendre la réalité sociale, s'y situer, décider de quelle manière procéder et de justifier ses comportements dans son contexte social. Or, la structure tripartite du concept de représentation sociale (valeurs, idées, pratiques) rappelle beaucoup celle d'attitudes (dimensions cognitive, affective et conative). Aussi, les deux concepts supposent-ils une sorte de réaction évaluative à l'égard d'un objet d'intérêt ${ }^{4}$. Cependant, on peut observer quelques différences importantes entre ces deux concepts 5 . En premier lieu, les représentations sociales s'appuient sur les comportements verbaux et manifestes, étant plus faciles à aborder et à traiter que les

2. L'incompatibilité entre les attitudes et le comportement est un thème débattu dans la psychologie sociale dès le milieu du XXe siècle (voir p. ex. Ajzen, 2005).

3. Voir aussi : « [...] a social representation is the ensemble of thoughts and feelings being expressed in verbal and overt behaviour of actors which constitutes an object for a social group » (Wagner et al., 1999: 95).

4. Rappelons ici l'une des définitions les plus répandues du concept d'attitude : « [...] a disposition to respond favorably or unfavorably to an object, person, institution, or event » (Ajzen, $2005: 3$ ).

5. La préoccupation méthodologique pour le rapport entre les attitudes et les représentations sociales a fait objet de nombreuses réflexions dans le cadre de la psychologie sociale (Jaspars et Fraser, 1984 ; Doise, 1989 ; Farr, 1994 ; Ruoquette et Rateau, 1998 ; Howarth, 2006, etc.). Pour une mise au point récente, voir p. ex. Salès-Wuillemin, Stewart et Dautun, 2004. 
attitudes. Ensuite, elles appartiennent à la société, non pas à l'individu. Finalement, et c'est le plus important, la théorie de la représentation sociale part d'une idée ancrée dans le constructivisme social, selon laquelle il n'y a pas de réalité sociale hors des représentations construites par le groupe social; un objet social coïncide avec les représentations sociales que l'on se fait de cet objet. Les différences mentionnées relèvent du fait que ces deux concepts - les attitudes et les représentations sociales - sont issus de deux paradigmes philosophiques complètement opposés et qu'ils sont incompatibles du point de vue épistémologique (Farr, 1994 ; Howarth, 2006 : 18)..

Notre adhésion au concept de représentation sociale n'est pas due seulement au cadre théorique commun du projet dans lequel s'inscrit notre recherche. Elle relève aussi de notre conviction que ce concept édifiant s'adapte mieux à l'étude d'une langue en danger et d'une communauté linguistique engagée sur la voie de la substitution linguistique. Ceci nous semble encore plus évident dans le cas de notre objet d'étude, la langue arbënishtë - source importante d'identification pour les membres de la communauté et pourtant caractérisée par un manque accentué de visibilité (Vuletić, 2014).

\section{La communauté arbënishtë : langue et identité}

Nous nous limitons à exposer ici quelques notions élémentaires concernant la situation historique et actuelle de l'arbënishtë, d'une importance essentielle pour comprendre la construction et la reconstruction des représentations sociales à l'intérieur de la communauté arbënishtë, aussi bien qu'en dehors de celle-ci. Variante de l'albanais guègue, l'arbënishtë est la langue des descendants des Albanais catholiques, originaires des villages de la région du lac de Shkodër, qui se sont installés, entre 1726 et 1754, dans trois localités de la périphérie de Zadar. Jouissant de la protection de l'archevêque de Zadar, ils ont été accueillis par les autorités vénitiennes qui leur donnèrent terres et cabanes?. Tandis que la communauté albanophone dans les localités de Zemunik et Ploče a subi une croatisation linguistique assez précoce, selon toute vraisemblance, la langue s'est maintenue jusqu'à nos jours à Arbanasi, aujourd'hui un quartier de la ville de Zadar et autrefois une localité rurale dans la banlieue de l'ancienne capitale de la Dalmatie vénitienne. L'intégration physique de la localité d'Arbanasi à la ville de Zadar est d'une date plutôt récente : elle ne se donne comme fait accompli qu'après la Seconde Guerre mondiale.

L’arbënishtë a fait objet de quelques études linguistiques importantes, comme celles de Tagliavini (1937) ou encore d'Ajeti (1961), mais jamais d'une étude sociolinguistique. Première langue d'une grande majorité de presque 2900 habitants du quartier d'Arbanasi, avant la Seconde Guerre mon-

6. Il est vrai qu'il existe des conceptualisations assez différentes (représentations sociales) des attitudes, et que certaines d'entre elles ont une base moins individualiste que d'autres (et qu'il y a des auteurs qui, pour cette raison, préfèrent le terme d'attitudes sociales).

7. Parmi les nombreuses raisons de leurs migrations, on cite d'habitude une situation économique difficile, des épidémies, l'anarchie et la vendetta présentes dans cette région de frontière que leur ancienne patrie, ainsi que l'intolérance religieuse (Krstić, $1985: 5-7)$. 
diale, l'arbënishtë est aujourd'hui utilisé par environ 200 habitants de la ville, la plupart d'entre eux habitants de l'ancien quartier et âgés entre 60 et $90 a^{8} s^{8}$. Cette diminution rapide du nombre de locuteurs est une conséquence non seulement d'une émigration de masse dans les années 50 du XXe siècle (Stipčević, 2011 : 19), mais aussi de l'abandon de l'arbënishtë par ceux restés en ville. Surtout dans les années 50 et 60, la communauté arbënishtë a été stigmatisée par les autorités communistes. Reléguée de plus en plus à l'usage familial, l'arbënishtë a subi, dans l'espace de 30 ans environ, la rupture définitive de la transmission générationnelle de la langue : aujourd'hui, il n'y a plus qu'une seule famille à Zadar où tous les membres utilisent cette langue, les locuteurs au-dessous de 40 ans étant au nombre de quatre9. C'est une langue en voie d'extinction. La plupart des locuteurs de l'arbënishtë se caractérisent par un multilinguisme vernaculaire complexe qui inclut la variété vénitienne et la koiné croate de la ville, ainsi que l'ancien dialecte croate tchakavien chez les locuteurs les plus âgés. À cela s'ajoutent, au niveau de la langue standard, le croate et, bien souvent, l'italien.

L'arbënishtë n'a jamais eu accès aux institutions publiques. À l'époque du bilinguisme officiel dans la ville, dans les dernières années de l'Empire austro-hongrois, l'école publique croate du quartier, ainsi que l'école privée italienne, offraient aux intéressés des cours d'albanais (Stipčević, 2011 : 224226), non pas d'arbënishtë. Cette ouverture se termina en 1920, quand la ville de Zadar est incorporée à l'Italie, victorieuse dans la Grande guerre. Toute institution scolaire non-italienne fut abolie par les autorités fascistes en 1923, et l'éducation en croate ne sera rétablie qu'en 1944, suite à l'entrée des partisans en ville. Durant cette période, des générations entières d'Arbënishtës ont été scolarisées exclusivement en italien. Dans l'après-guerre, leur croate imparfait a souvent été objet de moqueries, faits mentionnés par tous nos témoins. L'actuelle école du quartier, avec le croate comme seule langue véhiculaire de l'enseignement, organise parfois des activités de prise de conscience de l'arbënishtë, mais c'est là où tout s'arrête. Les cours libres de l'arbënishtë, organisés par une association locale en 2010, n'ont pas su susciter l'intérêt des jeunes (Kolbas, 2011 : 58).

En dépit de ces tendances négatives, il faut souligner que l'identité arbënishtë se caractérise par une vitalité étonnante et une évidente capacité de recréation, qui semble ne pas dépendre de la transmission de la langue ancestrale, mais plutôt des liens communautaires. Le nombre d'habitants de Zadar prêts à se déclarer comme Arbanasi 'Arbënishtës' pourrait facilement dépasser jusqu'à dix fois le nombre de locuteurs de l'arbënishtë. Les études manquent sur ce phénomène bien connu des habitants de Zadar (y compris les auteurs de cette contribution). Néanmoins, nous sommes en mesure d'affirmer que l'identité arbënishtë relève en premier lieu des origines arbënishtë de l'individu : l'appartenance à une famille que l'on sait historiquement arbënishtë suffit pour qu'on puisse incontestablement s'identifier comme Arbënishtë. Bien que formée à partir d'un critère aussi conser-

8. Cette situation sociolinguistique est reflétée aussi par notre échantillon : notre groupe de locuteurs inclut les personnes âgées entre 50 et 90 ans. Aux chiffres cités il faudrait ajouter les locuteurs résidant ailleurs en Croatie, notamment dans la capitale, ainsi que les émigrés, surtout en Italie. Kovačec parle d’un maximum de 500 locuteurs (Kovačec, 2002 : 68).

9. Il s’agit de trois frères et de leur cousin. Le locuteur le plus jeune est né en 1985. 
vateur, l'identité arbënishtë n'est pas imperméable. De nombreux noms de familles témoignent d'un nombre considérable d'hommes provenant des villages croatophones des alentours qui ont fondé des familles avec des femmes albanophones du quartier. Le cas des Bajlo est particulièrement instructif. Les porteurs de ce nom de famille, originaires d'une île voisine, se sont installés à l'extrémité de la presqu'île (Punta Bajlo) occupée aujourd'hui par le quartier d'Arbanasi, à peu près à l'époque où les réfugiés albanais arrivent à Zadar (Krstić, 1987: 11). À l'origine une enclave croatophone au sein de l'enclave albanophone, les Bajlo sont considérés comme des « Arbënishtës de souche » à tous les sens du terme.

\section{Mise en place du questionnaire et conduite des enquêtes}

Les groupes sociaux se distinguent mutuellement selon la manière de comprendre un phénomène social donné, laquelle à son tour constitue leur identité sociale. Cette compréhension partagée est le fondement de la communication et des autres formes de coaction ${ }^{10}$. Une partie au moins de la compréhension partagée par les membres d'un groupe social donné doit être différente de la compréhension des non-membres. Dans le cas contraire, il n'y aurait pas beaucoup de sens à parler des groupes sociaux. À partir de cette idée, nous avons porté à terme trois enquêtes relatives aux représentations de la langue arbënishtë, auprès de trois groupes sociaux différents :

- locuteurs d'arbënishtë, femmes et hommes, au-dessus de 50 ans, tous nés à Zadar et tous habitants de la ville (mais pas tous de l'ancien quartier arbënishtë);

- étudiantes en langues et littératures (philologies allemande, anglaise, catalane, croate, portugaise, russe) à l'Université de Zadar, âgées entre 19 et 22 ans, toutes nées et toutes résidant à Zadar, d’origine non-arbënishtë, de langue maternelle croate, non-locutrices de l'arbënishtë ;

- élèves, des deux sexes, des classes terminales de l'école primaire du quartier arbënishtë, âgés entre 13 et 14 ans, tous nés et tous résidant à Zadar, de langue maternelle croate, non-locuteurs de l'arbënishtë, tous se définissant eux-mêmes comme Arbanasi 'Arbënishtës', voire 'membres de la communauté arbënishtë'.

En ce qui concerne la composition de nos échantillons, les locuteurs étaient au nombre de vingt, les étudiantes au nombre de quarante, tandis que les élèves étaient au nombre de dix-sept $\mathrm{t}^{11}$. Les trois échantillons ont un haut dégrée de représentativité : le premier arrive à environ $10 \%$ du nombre total de locuteurs de l'arbënishtë dans la ville ; le second représente 2,5\% d'étudiants de l'Université de Zadar ; le troisième arrive à 2,8 \% d'élèves de l'école du quartier.

10. Ce sont justement les représentations sociales qui « reflètent la recherche d'une compréhension socialement partagée » (Fiske, 2008 : 659).

11. Vingt-cinq élèves des classes terminales de cette école se disent Arbënishtës, indépendamment de leurs origines, mais seulement dix-sept questionnaires ont été renseignés de manière correcte. Parmi cinquante-neuf élèves des classes terminales qui ne se définissent pas comme Arbënishtës, il y en a qui sont effectivement d'origine arbënishtë. 
Dans l'élaboration des questionnaires, ainsi que dans la conduite des enquêtes, nous avons rigoureusement respecté le cadre méthodo logique du projet dans lequel s'inscrit notre recherche (Maurer, 2013 : 50-52 ; 63-65). Dans chacune des trois enquêtes, nous avons utilisé un questionnaire différent, dont la composition relève des éléments de discours épilinguistique sur la langue arbënishtë, recueillis auprès de trois groupes différents de témoins des mêmes caractéristiques démographiques et sociolinguistiques que celles de l'échantillon relatif:

- pour l'enquête avec les locuteurs, 20 voisins, locuteurs de l'arbënishtë;

- pour l'enquête avec les étudiantes, 20 camarades ;

- pour l'enquête avec les élèves, 76 camarades.

Les sujets étaient invités à s'exprimer, à l'oral dans le premier groupe et à l'écrit dans deux autres groupes, sur les idées qu'évoquait pour eux la locution « la langue arbënishtë». À partir des éléments de discours les plus fréquents dans chaque groupe de témoins, trois questionnaires ont été constitués et soumis aux membres de trois échantillons relatifs ${ }^{12}$. Le nombre différent d'items dans chaque questionnaire renvoie directement à l'enthousiasme avec lequel chaque groupe de témoins a répondu à notre appe ${ }^{13}$. Le questionnaire reparti dans le groupe de locuteurs de l'arbënishtë comportait 20 items, celui utilisé avec les étudiantes 15 items, tandis que celui passé aux élèves était composé de 10 items. Dans ce qui suit, nous présentons la structure des trois questionnaires.

\section{(1) Questionnaire locuteurs}

1. Une langue à laquelle je suis particulièrement attaché(e).

2. Je ne perds pas l'opportunité de la parler.

3. Je ne la parle qu'avec les membres de ma famille et avec mes amis les plus proches.

4. Une langue qui rend possible la communication avec quelques Albanais.

5. Une langue des vieux.

6. La langue de mon enfance.

7. Il est dommage que ce ne soit pas une langue écrite.

8. Une langue qui meurt.

9. Je suis fier de la parler encore.

10. On se sent inférieur quand on le parle.

12. Les personnes qui auparavant avaient participé dans les groupes de témoins n'ont pas été inclues dans l'échantillon. 13. La volonté de partager les réflexions sur sa propre langue a été très prononcée dans le groupe de témoins composé par les locuteurs de l'arbënishtë. 
11. On se sent bien quand on le parle.

12. Je ne l'utilise pas en public.

13. On doit le mélanger avec le croate ou l'italien si on veut bien s'exprimer.

14. Une langue où j'arrive mieux à dire ce que je pense.

15. Les tentatives de le sauver sont vaines.

16. Je suis désolé(e) de le voir disparaitre.

17. Une langue qui ne sert à rien.

18. Il faudrait l'introduire à l'école primaire du quartier.

19. Il faudrait le parler aux enfants.

20. C'est de notre faute si les jeunes ne le parlent pas.

\section{(2) Questionnaire étudiants}

1. La langue des Albanais.

2. Ça sonne italien.

3. Une langue dont on ne sait pas assez à Zadar.

4. Une langue qui meurt.

5. Les locuteurs de l'arbënishtë ont honte de leur langue.

6. Une langue qu'on ne peut pas sauver.

7. Une langue qu'il faudrait protéger.

8. Une langue que j'aimerais bien comprendre.

9. Une partie importante du patrimoine de Zadar.

10. L’arbënishtë devrait être une matière optionnelle dans les écoles de Zadar.

11. Il faudrait que l'arbënishtë soit plus visible dans l'espace public de Zadar.

12. Il faudrait que les locuteurs de l'arbënishtë le parlent avec leurs enfants.

13. Une langue qui ne sert à rien.

14. Une langue qui ne m'intéresse pas vraiment.

15. Il faudrait en parler plus dans les écoles de Zadar. 


\section{(3) Questionnaire élèves}

1. Une langue des vieux.

2. Ça sonne italien.

3. Une belle langue.

4. Une langue étrange.

5. La langue parlée par les gens du quartier d'Arbanasi.

6. Une langue ennuyeuse.

7. Une langue traditionnelle.

8. La langue de mes anciens.

9. La langue que parlent mes amis.

10. C'est de l'albanais.

Lors de la première enquête, nous nous sommes aperçus que le système de notes $-2,-1,0,+1,+2$ (où +2 marque le plus fort degré d'adhésion, -2 le plus fort rejet), à utiliser dans la valuation des items, ne fonctionnait pas très bien dans le contexte croate. La cause principale en est qu'il rappelle trop les notes les plus basses du système d'éducation croate, difficiles de mettre en relation avec des idées positives (qui ne manquaient pas dans le questionnaire). C'est pour cela que pour l'usage de nos témoins ce système a été transposé à celui de 1 à 5 (où 5 marque le plus fort degré d'adhésion, 1 le plus fort rejet). Lors du traitement postérieur des résultats, leurs réponses étaient codées de manière suivante : $1=-2,2=-1,3=0,4=+1,5=+2$.

Les membres de chaque échantillon ont reçu les instructions relatives au renseignement du questionnaire (Maurer, 2013 : 52), adaptées au système de notes de 1 à 5. Ils étaient donc invités:

- d'abord à noter 5 les propositions qui paraissent le mieux évoquer l'arbënishtë ;

- $\quad$ puis de noter 1 les propositions qui paraissent le moins bien évoquer l'arbënishtë ;

- de noter 4 les propositions qui paraissent assez bien évoquer l’arbënishtë ;

- de noter 2 les propositions qui paraissent assez mal évoquer l'arbënishtë ;

- de noter 3 les propositions restantes. 
En fonction du nombre d'items dans le questionnaire, les sujets étaient obligés d'opérer des groupements et d'utiliser chaque note:

- quatre fois exactement dans le questionnaire à 20 items ;

- trois fois exactement dans le questionnaire à 15 items;

- deux fois exactement dans le questionnaire à 10 items.

Les questionnaires étaient administrés en croate. La conduite des enquêtes avec les étudiants ne présentait pas de difficultés particulières. De vingt-cinq élèves des classes terminales dans l'école du quartier, se définissant comme Arbënishtës, dix-sept seulement ont renseigné le questionnaire de manière correcte. Quant aux locuteurs de l'arbënishtë, quelques sujets les plus âgés avaient du mal à renseigner tout seuls le questionnaire, à cause de leur maîtrise imparfaite du croate standard. Dans ces cas, nous étions obligés de leur traduire en vénitien ou en arbënishtë les propositions qu'ils n'entendaient pas bien ${ }^{14}$.

\section{L’analyse des données}

Notre analyse est conçue comme présentation et interprétation du score d'adhésion ou de rejet du groupe par rapport aux items du questionnaire relatif, et de l'indice de consensus des sujets pour chacun de nos trois groupes de témoins ${ }^{15}$. On entend par « score » la moyenne de l'ensemble des valuations des individus formant le groupe, les scores maximums possibles étant -2 et +2 (Maurer, 2013: 53). Le calcul de l'indice du consensus mesure les écarts des notations individuelles aboutissant au score moyen (Maurer, 2013 : 67). Ces résultats sont ensuite visualisés sous forme d'un graphe de la représentation, voire d'un schéma en couronnes de la structure de la représentation sociale. Sur le graphe, l'adhésion est numérotée de -2.00 à 2.00 sur la ligne horizontale (la plus basse à gauche, la plus grande à droite), tandis que le consensus est indiqué par la taille des cercles. La position verticale des cercles ne reflète aucune valeur. Le schéma en couronnes reflète l'intégration adhésion-consensus, avec quatre zones où les cognèmes sont repartis en fonction du score d'adhésion et de l'indice de consensus, le seuil de 0,07 retenu comme discriminant entre le traitement consensuel et le traitement dispersé (v. Maurer, 2013: 69). II va de soi que les résultats quantitatifs requièrent une interprétation à la lumière d'un ensemble de macro- et micro-facteurs sociohistoriques et sociolinguistiques.

14. On tient à remercier Mme Maximiljana Barančić pour sa participation et son aide au cours des enquêtes.

15. Le score d'adhésion et l'indice de consensus sont automatiquement calculés sur un tableur disponible sur le site http://linguiste.iutbeziers.fr. 


\subsection{Première enquête : représentations de l'arbënishtë par les locuteurs de cette langue}

Tableau 1 : Calcul du score d'adhésion et de l'indice de consensus (enquête locuteurs)

\begin{tabular}{|c|c|c|c|c|c|c|c|c|c|c|}
\hline Item & 1 & 2 & 3 & 4 & 5 & 6 & 7 & 8 & 9 & 10 \\
\hline Score d'adhésion & 1.15 & 0.85 & 0.5 & -1.35 & 0.6 & 1.65 & -0.1 & 0.05 & 1.3 & -2 \\
\hline $\begin{array}{c}\text { Indice de } \\
\text { consensus }\end{array}$ & 0.084 & 0.087 & 0.083 & 0.11 & 0.089 & 0.117 & 0.091 & 0.072 & 0.094 & 0.5 \\
\hline Item & 11 & 12 & 13 & 14 & 15 & 16 & 17 & 18 & 19 & 20 \\
\hline Score d'adhésion & 0.3 & -0.5 & 0.35 & -1.3 & -0.75 & 0.75 & -1.85 & -1.05 & -0.1 & 1.3 \\
\hline $\begin{array}{c}\text { Indice de } \\
\text { consensus }\end{array}$ & 0.119 & 0.102 & 0.11 & 0.104 & 0.077 & 0.088 & 0.192 & 0.093 & 0.088 & 0.099 \\
\hline
\end{tabular}

Un nombre plus grand d'items dans le questionnaire que nous avons utilisé dans l'enquête avec les locuteurs de l'arbënishtë fait que le score d'adhésion soit bien distribué (de -2.00 à 1.78). Cependant, le consensus est très bas pour chaque item, à cause d'une grande dispersion dans les réponses (Figure 1). La seule exception représente l'item 10 ("On se sent inférieur quand on le parle »), rejeté par une grande majorité de nos témoins. La valuation positive des items situés à droite du schéma indique que les locuteurs de l'arbënishtë se sentent attachés à leur langue d'enfance (6) et que la plupart d'eux sont fiers de la parler $(9,11)$. Ils sont en même temps conscients que l'arbënishtë est une langue en voie de disparition (8). L'adhésion à plusieurs items confirme ce point de vue. Pour tous les sujets, l'arbënishtë est une langue réservée au domaine privé (3 et 12). Bien que beaucoup de répondants affirment parler l'arbënishtë le plus souvent possible (2), la plupart d'entre eux se rendent compte de la leaking diglossia : ils avouent que leur compétence en arbënishtë est plus basse qu'en d'autres langues qu'ils maitrisent (14), avec une forte incidence de code-mixing (13) avec les adstrats croate et italien ${ }^{16}$. Ces deux facteurs sont typiques du processus de la substitution linguistique (Dorian, 1981 ; Schmidt, 1985 ; etc.). Les locuteurs âgés de l'arbënishtë sont « désolés de le voir disparaitre » (16). Cependant, lorsqu'ils se reconnaissent responsables pour la rupture de la transmission générationnelle de la langue (20), ils sont bien indécis devant la possibilité de la parler aux enfants (19), et décidément pas en faveur de l'introduire à l'école primaire (18). Les données du Tableau 1 montrent que le pessimisme est omniprésent parmi les locuteurs de l'arbënishtë (15), ce qui entraine les scores bas des aspects comportementaux de leurs affirmations, malgré un haut niveau d'attachement affectif à la langue, ainsi que une conscience cognitive de la réalité sociolinguistique actuelle.

L'intégration adhésion-consensus montre que la structure de la représentation de l'arbënishtë chez les locuteurs de cette langue (Figure 2) est organisée autour d'un ensemble composé de quatre éléments : le sentiment d'un attachement particulier à la langue (1), langue à travers laquelle ils maintiennent un lien avec leur enfance (6), la fierté de la parler (9), malgré une longue expérience de

16. Avec le terme italien, nos témoins désignent le plus souvent la variété vénitienne locale. 
stigmatisation, ainsi que le regret de ne l'avoir pas transmise aux jeunes (20). Ce sont les éléments centraux de l'autoreprésentation de la communauté linguistique arbënishtë.

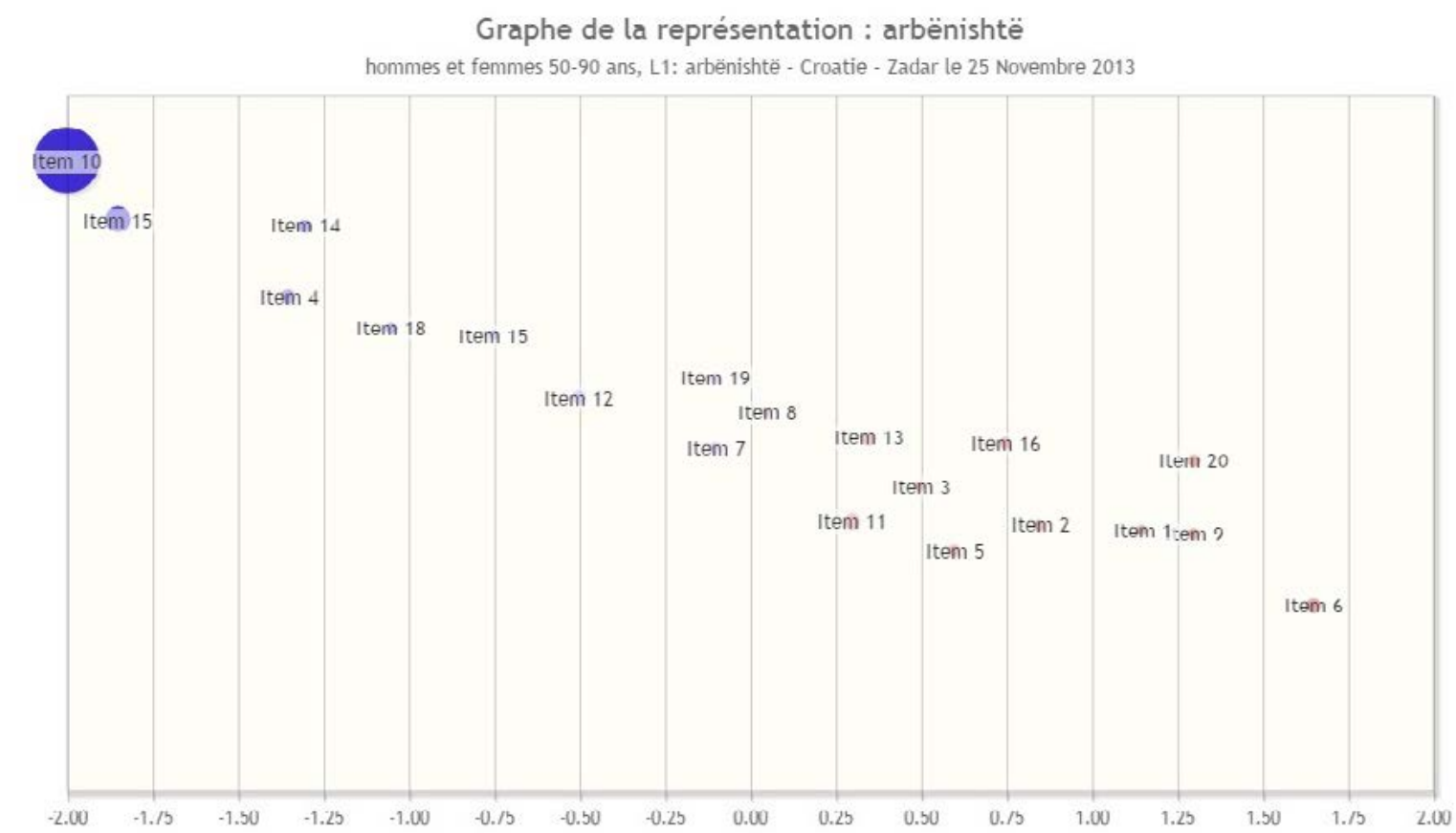

Figure 1 : Graphe de la représentation (enquête locuteurs)

Schéma en couronnes de la structure de la représentation sociale : arbënishtë

hommes et femmes 50-90 ans, L1: arbënishtë le 25 Novemb

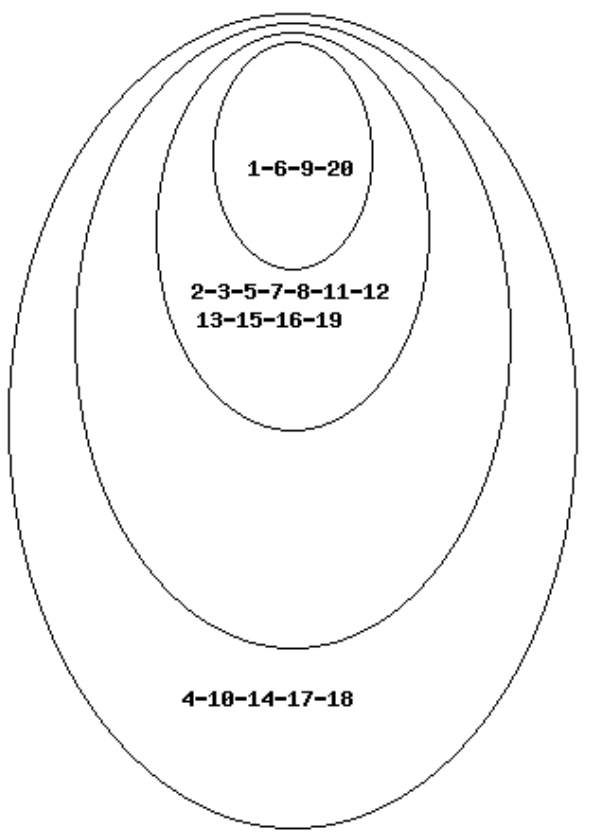

Figure 2 : Structure de la représentation (enquête locuteurs) 
5.2. Deuxième enquête : représentations de l'arbënishtë par les non-locuteurs externes au groupe

Tableau 2: Calcul du score d'adhésion et de l'indice de consensus (enquête étudiantes)

\begin{tabular}{|c|c|c|c|c|c|c|c|c|}
\hline Item & $\mathbf{1}$ & $\mathbf{2}$ & $\mathbf{3}$ & $\mathbf{4}$ & $\mathbf{5}$ & $\mathbf{6}$ & $\mathbf{7}$ & $\mathbf{8}$ \\
\hline Score d'adhésion & -0.38 & 0.25 & 1.1 & 0.7 & -1.08 & -1.03 & 0.7 & -0.18 \\
\hline Indice de consensus & 0.062 & 0.068 & 0.089 & 0.076 & 0.099 & 0.08 & 0.078 & 0.089 \\
\hline Item & 9 & 10 & 11 & 12 & 13 & 14 & 15 & \\
\hline Score d'adhésion & 1.23 & -1.08 & -0.13 & 1.28 & -0.78 & -0.15 & -0.08 & \\
\hline Indice de consensus & 0.087 & 0.096 & 0.091 & 0.105 & 0.074 & 0.069 & 0.081 & \\
\hline
\end{tabular}

Ce groupe est composé par les étudiantes de langues modernes de l'Université de Zadar, non-locutrices de l'arbënishtë et d'origine non-arbënishtë, toutes nées à Zadar et habitantes de cette ville. Nous les avons supposées plus sensibles aux thèmes linguistiques, y compris la situation des langues minoritaires ou en voie de disparition, que les étudiants des programmes non-philologiques.

Le score d'adhésion et l'indice de consensus pour chaque item du questionnaire sont rapportés dans le tableau 2 et visuellement présentés sur le graphe et le schéma correspondants. Les réponses des étudiantes sont moins dispersées que celles de la première enquête, menée auprès des locuteurs de l'arbënishtë. Une taille plus grande des cercles (Figure 3 ) indique un consensus plus fort sur chaque item, tandis que le schéma en couronnes de la structure de la représentation sociale est plus équilibré dans ce cas (Figure 4). Les deux caractéristiques renvoient à une approche plus circonspecte auprès des étudiantes, ainsi qu'à une tendance à la neutralité à l'heure d'évaluer les items du questionnaire. Les raisons de cette tendance sont probablement à chercher dans un manque d'informations sur la langue et la communauté arbënishtës, ainsi que dans une attitude généralement démocratique vis-à-vis de la diversité linguistique locale.

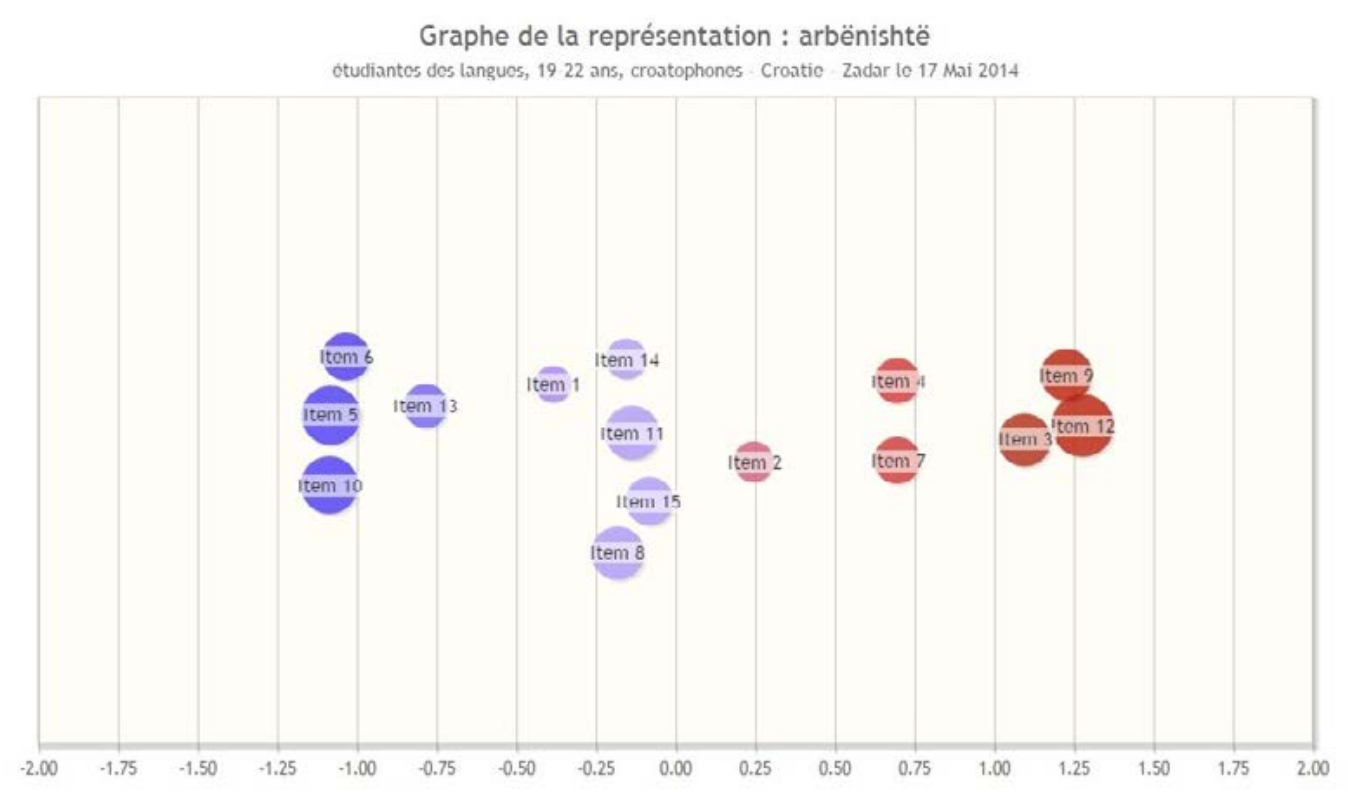

Figure 3 : Graphe de la représentation (enquête étudiantes) 
Les items situés à droite dans le graphe de la représentation (Figure 3) indiquent que les non-locuteurs de l'arbënishtë ayant reçu une éducation philologique, reconnaissent la valeur historique et culturelle de cette langue dans le contexte local (9). Ils considèrent l'arbënishtë comme une langue en voie de disparition (4), mais ils se montrent favorables à sa protection (7). Leurs réponses indiquent, néanmoins, qu'ils pensent plutôt à une promotion passive de l'arbënishtë (3), qu'ils laissent à la volonté des locuteurs (12) ${ }^{17}$. Une approche proactive, qui consisterait à introduire l'arbënishtë dans les écoles de Zadar en tant que matière optionnelle (10) ou à augmenter sa visibilité dans l'espace public de la ville (11), ne semble pas intéresser les membres de ce groupe. Ces attitudes concordent avec leur valuation des items d'orientation personnelle : l'arbënishtë est une langue qui les intéresse jusqu'à un certain point (14), mais non pas une langue qu'ils aimeraient comprendre (8).

Le schéma en couronnes de la structure de la représentation sociale (Figure 4) accentue le caractère central des aspects cognitifs des attitudes sociales à l'égard de la langue arbënishtë dans ce groupe $(3,9,12)$. À côté de ces trois représentations les plus stables, un ensemble de six items dans la couronne centrale $(4,7,8,11,13,15)$, avec des indices de consensus significatifs, montre une disposition généralement positive à l'égard de l'arbënishtë. À la différence du groupe d'items dans la zone de centralité maximum, cette zone rassemble un groupe plutôt varié de cognèmes qui se rapportent aux différents aspects d'attitudes, tels que convictions (4), dimensions affectives d'ordre plus personnel (p. ex. 8) ou dispositions d'entreprendre certaines actions (p. ex. 7, 11, 15). Les items dans la périphérie incertaine $(1,2,14)$, rassemblant les éléments de moindre importance, relèvent d'un manque d'informations sur l'objet de la représentation. Trois éléments se trouvent à la périphérie marginale $(5,6,10)$, dont deux $(5,10)$ font l'objet du consensus le plus fort.

17. L'indice de consensus sur l'item 12 est particulièrement intéressant, puisqu'il montre que les répondants ne connaissent pas la structure démographique de la communauté linguistique arbënishtë. 

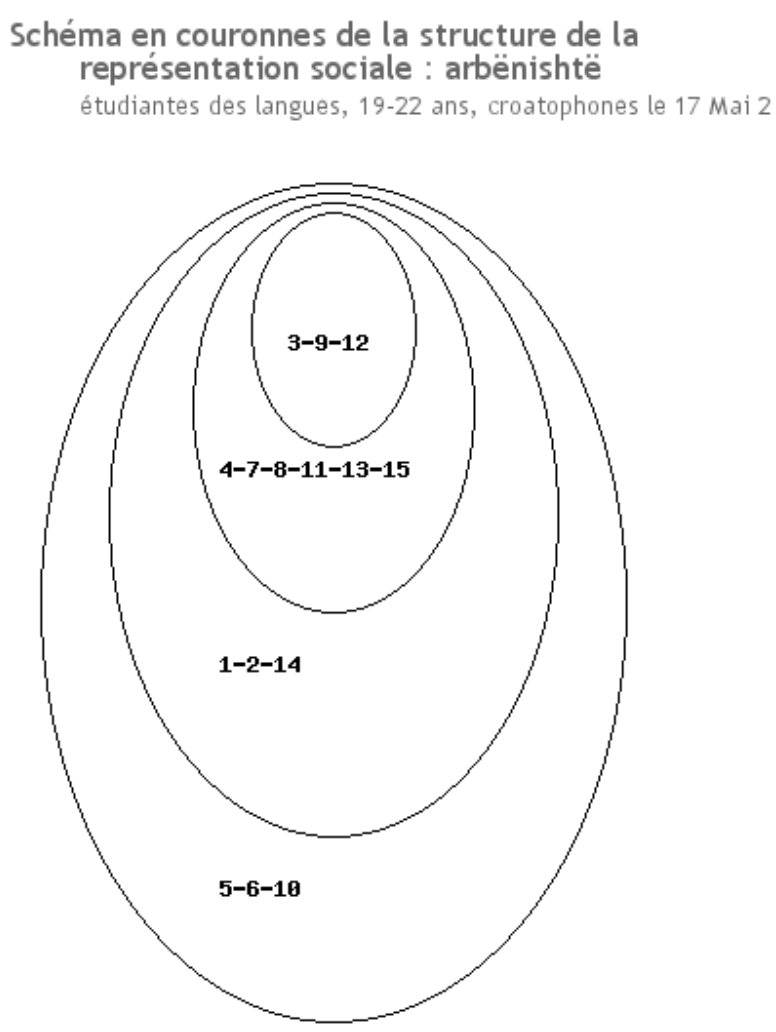

Figure 4 : Structure de la représentation (enquête étudiantes)

\subsection{Troisième enquête : représentations de l'arbënishtë par les non-locuteurs membres du groupe}

Tableau 3 : Calcul du score d'adhésion et de l'indice de consensus (enquête élèves)

\begin{tabular}{|c|c|c|c|c|c|c|c|c|c|c|}
\hline Item & 1 & 2 & 3 & 4 & 5 & 6 & 7 & 8 & 9 & 10 \\
\hline Score d'adhésion & 0.82 & -0.29 & 0.53 & -0.06 & 0.47 & -1.29 & 0.88 & 0.41 & -1.47 & 0 \\
\hline Indice de consensus & 0.073 & 0.074 & 0.066 & 0.073 & 0.078 & 0.106 & 0.105 & 0.076 & 0.134 & 0.066 \\
\hline
\end{tabular}

Notre troisième groupe est composé par les élèves des classes terminales de l'école primaire du quartier d'Arbanasi, non-locuteurs de la langue, mais qui s'identifient eux-mêmes comme Arbanasi «Arbënishtës ». Ils sont, donc, membres de la communauté traditionnelle arbënishtë, mais non pas de la communauté linguistique arbënishtë. Il est intéressant de noter que 12,5 \% d'élèves de cette école qui s'identifient comme Arbanasi ne sont pas d'accord avec la proposition « L'arbënishtë est la langue de mes anciens ». Cela implique la présence des élèves qui ont acquis l'identité arbënishtë, mais qui proviennent des familles historiquement non-arbënishtës, installées dans le quartier à une époque plus récente, aussi bien que celle des enfants provenant des familles historiquement arbënishtës qui ne se rappellent pas d'avoir entendu parler cette langue dans leurs familles respectives. 
Il sembley avoir peu de consensus entre les membres de notre échantillon sur presque tous les items du questionnaire (Tableau 3). Néanmoins, nous notons trois exceptions : l'arbënishtë est considéré comme une langue traditionnelle (7), ce qui rend impossible que ce soit une langue parlée par des amis (9), et ce n'est décidément pas une langue ennuyeuse (6). Le graphe de la représentation (Figure 5) montre un groupement très clair d'items : une telle différentiation nette ne se trouve pas auprès des deux premiers groupes, les locuteurs de l'arbënishtë et les étudiants non-locuteurs. Tandis que deux cognèmes ( 6 et 9) sont fortement repoussés, le score positif d'adhésion aux propositions relevant des convictions $(1,5,8)$ ou d'une évaluation personnelle $(3,7)$ est plus modeste. Un troisième groupe occupe la zone centrale du graphe, en indiquant un manque d'engagement personnel ou de connaissance sur l'arbënishtë. À titre d'exemple, le niveau d'incertitude en ce qui concerne les origines de la langue arbënishtë est observable autant chez les élèves (10), que chez les étudiants (1). Il n'y a pas beaucoup d'accord non plus sur la prétendue « italianité » des sons de l'arbënishtë: auprès des deux groupes, le score d'adhésion est presque 0 ( 0.25 chez les étudiants, -0.29 chez les élèves).

L'analyse de la structure de la représentation (Figure 6) montre une zone de centralité maximum vide, avec la majorité de cognèmes concentrés dans la couronne centrale. Cependant, cet ensemble ne contient qu'une proposition évaluative (" une langue étrange »), tandis que les autres (1, 2, 5, 7, 8) relèvent de la connaissance (ou du manque de celle-là). D'une façon semblable, on trouve une proposition évaluative dans la périphérie incertaine (3) et une dans la périphérie marginale (6).

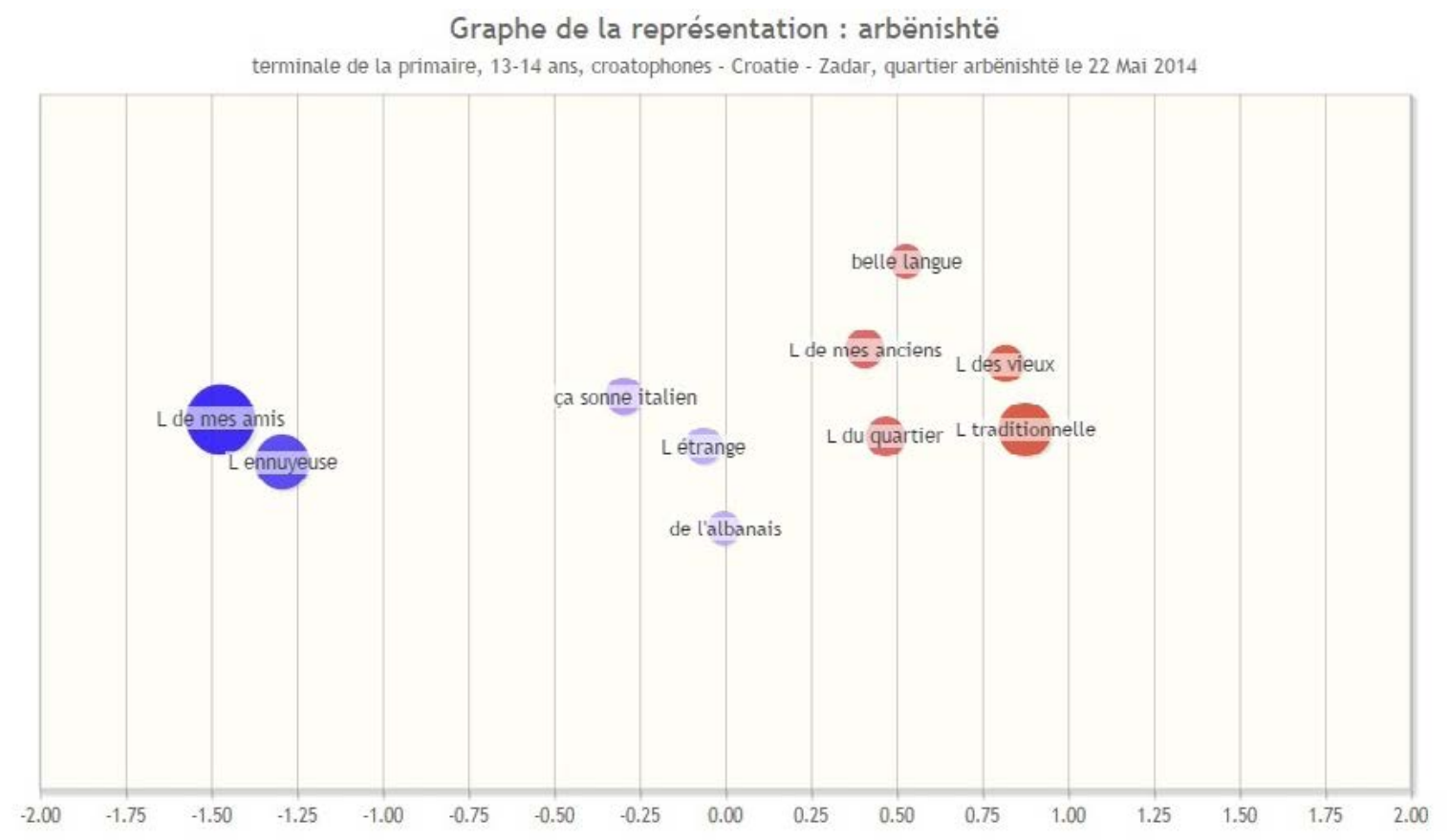

Figure 5 : Graphe de la représentation (enquête élèves) 

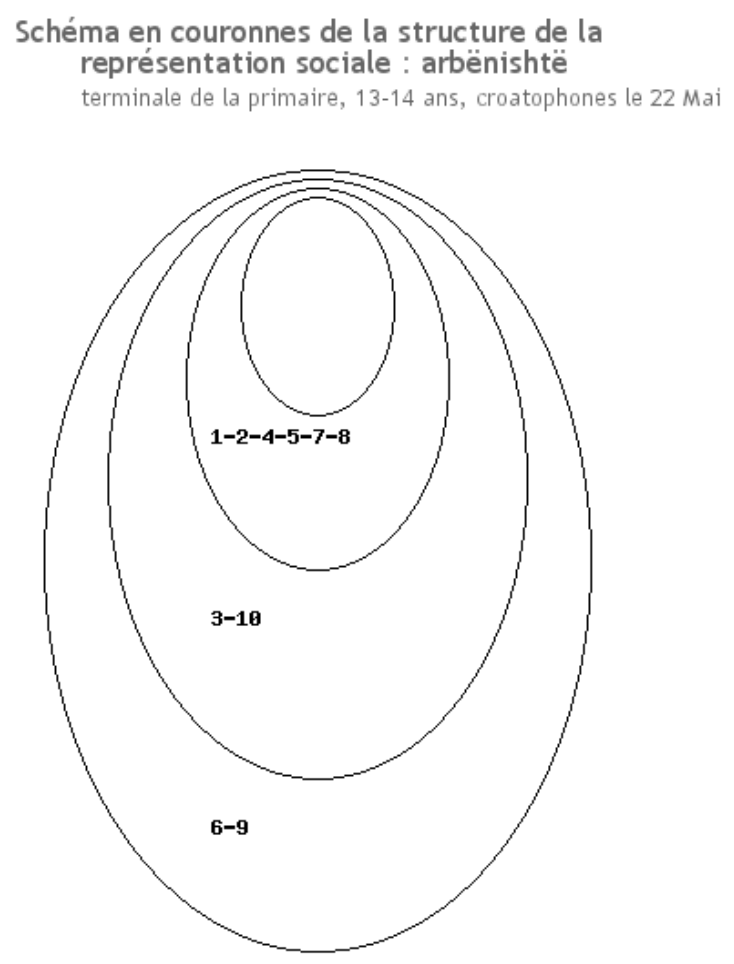

Figure 6 : Structure de la représentation (enquête élèves)

\section{Discussion et conclusion}

Les hauts niveaux de dispersion des représentations et les niveaux bas de consensus dans le groupe constitué par les locuteurs de l'arbënishtë sont déjà indicatifs du statut et du prestige de cette langue parmi les membres de la communauté linguistique arbënishtë. Tandis que ces deux éléments sont présents de manière implicite, l'attribution explicite des scores à certains items du questionnaire pourrait suggérer une conclusion différente.

Malgré leur conviction que l'arbënishtë est une langue en voie de disparition, les étudiants non-locuteurs sont autant optimistes que les locuteurs eux-mêmes, lorsqu'ils pensent que la langue peut être sauvée. Dans le même ordre d'idées, les répondants des deux groupes repoussent l'idée de l'inutilité de l'arbënishtë, ils sont désolés de le voir disparaître et croient qu'il faudrait le protéger. Chez les locuteurs, les remords provoqués par le processus évident et imminent de la disparition de cette langue, aussi bien que l'idée de sa protection, restent néanmoins assez vagues, à cause de l'attitude « là où vous voudrez mais pas chez moi » qui bloque toute action concrète dirigée contre le déclin de l'arbënishtë.

Une observation méthodologique intéressante s'impose : les questionnaires que nous avons pu construire à partir des éléments de discours des deux groupes de jeunes non-locuteurs - étudiantes et élèves respectivement - sont numériquement moins fournis et contiennent les cognitions moins personnelles que celui construit à partir des éléments de discours des locuteurs âgés, ce qui montre 
une attitude moins personnelle avec la langue objet de notre étude. II serait possible d'interpréter cette circonstance en fonction de l'âge de chaque groupe et, avec cela, mettre le nombre plus réduit de cognitions en relation avec une expérience plus humble ou une capacité plus modeste de verbaliser sa position personnelle vers la langue arbënishtë. Deux arguments au moins contredisent cette supposition. D’abord, notre deuxième groupe est composé par les étudiantes de langues et littératures : leur éducation devrait leur permettre de formuler des cognitions plus personnelles à l'égard de l'objet d'étude, dans le cas où ces cognitions feraient partie de leurs représentations sociales. En deuxième lieu, nos élèves sont membres de la communauté traditionnelle arbënishtë, ce qui devrait leur permettre un attachement plus personnel à leur langue ancestrale que celui indiqué par la structure du questionnaire. Certes, les divergences dans la structure de nos trois questionnaires pourraient relever aussi d'éventuelles erreurs méthodologiques de notre part, mais nous croyons qu'elles indiquent plutôt un détachement graduel à l'égard de l'arbënishtë et de sa communauté linguistique. Ce détachement est visible dans la synchronie, mais du point de vue diachronique il relève d'une lente démise des fonctions instrumentales et symboliques de la langue.

L'une des implications de cette étude c'est que la notion de représentation sociale s'adapte très bien à l'étude des communautés qui expérimentent un changement unidirectionnel, souvent vers la substitution linguistique, accompagnée par la perte de la langue. Cette idée est particulièrement applicable au contexte arbënishtë, étant donné qu'une communauté arbënishtë démographiquement, ethniquement et linguistiquement différenciée de son entourage n'existe plus. La langue ancestrale s'est conservée auprès d'une mince minorité de ceux qui se disent Arbënishtës. Il existe, en revanche, toute une communauté qui affirme une identité arbënishtë, en réclamant une tradition identifiée avec cet ethnonyme. Cette communauté, que nous avons nommée provisoirement communauté traditionnelle arbënishtë, est ancrée dans la représentation sociale et beaucoup plus nombreuse que la communauté linguistique arbënishtë. Il est important de souligner que la représentation sociale de la communauté expérimente un changement: chez les élèves s'identifiant comme Arbënishtës, il y a une tendance à étendre l'identité arbënishtë aux amis d'origine non-arbënishtë que vivent dans le quartier. Ainsi, notre étude nous a permis non seulement d'entrevoir la structure des représentations sociales de la langue arbënishtë, mais aussi le processus dynamique de la restructuration d'une identité collective. 


\section{Références}

Abric, Jean-Claude (1989), "L'étude expérimentale des représentations sociales », dans Denise Jodelet (dir.), Les représentations sociales, Paris, Presses universitaires de France, p. 187-203.

Abric, Jean-Claude (1994), «Les représentations sociales: aspects théoriques », dans Jean-Claude Abric (dir.), Pratiques sociales et représentations, Paris, Presses universitaires de France, p. 11-36.

Ajeti, Idriz (1961), Istorijski razvitak gegijskog govora Arbanasa kod Zadra, Sarajevo, Akademija nauka Bosne i Hercegovine.

Ajzen, Icek (2005), Attitudes, personality and behaviour, Maidenhead, Open University Press \& McGrawHill Education.

Bourhis, Richard Y. et Itesh Sachdev (1984), «Vitality perceptions and language attitudes : some Canadian data », Journal of Language and Social Psychology, vol. 3, n² 2, p. 97-126.

Boyer, Henri (1996), «Les domaines de la sociolinguistique », dans Henri Boyer (dir.), Sociolinguistique : territoire et objets, Lausanne, Delachaux et Niestlé, p. 9-34.

Bradley, David (2002), «Language attitudes : the key factor in language maintenance », dans David Bradley et Maya Bradley (dir.), Language Endangerment and Language Maintenance, London, Routledge Curzon, p. 1-10.

Doise, Wilhelm (1989), «Attitudes et représentations sociales », dans Denise Jodelet (dir.), Les représentations sociales, Paris, Presses universitaires de France, p. 220-237.

Dorian, Nancy (1981), Language Death : the Life Cycle of a Scottish Gaelic Dialect, Philadelphia, University of Pennsylvania Press.

Farr, Robert (1994), «Attitudes, social representations and social attitudes », Papers on social representations, vol. 3, nº 1, p. 30-33.

Fiske, Susan T. (2008), Psychologie sociale, Bruxelles, De Boeck. [Titre original : Social Beings : a core motives approach to social psychology, New York, Wiley, 2004. Traduit de l'anglais par Valerie Provost et Sophie Huyghues Despointes.]

Giles, Howard, Doreen Rosenthal et Louis Young (1985), "Perceived ethnolinguistic vitality : the Anglo- and Greek-Australian setting », Journal of Multilingual and Multicultural Development, $n^{\circ} 6$, p. 253-69.

Guimelli, Christian (1999), La pensée sociale, Paris, Presses universitaires de France.

Howarth, Caroline (2006), « How social representations of attitudes have informed attitude theories: the consensual and the reified », Theory and psychology, vol. 16, n 5, p. 691-714.

Jaspars, Jos et Colin Fraser (1984), «Attitudes and Social Representations », dans Robert M. Farr et Serge Moscovici (dir.), Social Representations, Cambridge, Cambridge University Press, p. 101-124. 
Kolbas, Irena (2011), « Dokumentiranje i muzealizacija ugroženih jezika Hrvatske », Etnološka istraživanja, no 16, p. 45-61.

Kovačec, August (2002), « Arbanasi-Albanisch », dans Miloš Okuka (dir.), Lexikon der Sprachen des Europäischen Ostens, Klagenfurt, Wieser, coll. «Wieser Enzyklopädie des Europäischen Ostens », vol. 10, p. 67-70.

Kristiansen, Tore et Jens Normann Jørgensen (2005), " Subjective factors in dialect convergence and divergence », dans Peter Auer, Frans Hinskens et Paul Kerswill (dir.), Dialect Change : the Convergence and Divergence of Dialects in Contemporary Societies, Cambridge, Cambridge University Press, p. 287-302.

Krstić, Kruno (1987), Rječnik govora zadarskih Arbanasa, Zadar, Mjesna zajednica Arbanasi.

Maurer, Bruno (2013), Représentations sociales des langues en situation multilingue : la méthode d'analyse combinée, nouvel outil d'enquête, Paris, Éditions des archives contemporaines.

Moscovici, Serge (1961), La psychanalyse : son image et son public, Paris, Presses universitaires de France.

Moscovici, Serge (1963), «Attitudes and opinions », Annual Review of Psychology, n 14, p. 231-260.

Moscovici, Serge (1973), "Foreword», dans Claudine Herzlich (dir.), Health and illness : a social psychological analysis, London/New York, Academic Press, p. ix-xiv.

Moscovici, Serge (1988), « Notes towards a Description of Social Representations », European Journal of Social Psychology, $n^{\circ} 18$, p. 211-250.

Rouquette, Michel-Louis et Patrick Rateau (1998), Introduction à l'étude des représentations sociales, Grenoble, Presses universitaires de Grenoble.

Salès-Wuillemin, Édith, Isobel Stewart et Marc Dautun (2004), «Effets de l'activation d'une représentation sociale par l'attitude : étude expérimentale », Cahiers Internationaux de Psychologie Sociale, nº 61, p. 43-56.

Sasse, Hans-Jürgen (1992), «Theory of language death », dans Matthias Brenzinger (dir.), Language Death: Factual and Theoretical Explorations with Special Reference to East Africa, Berlin/New York, Mouton de Gruyter, p. 7-30.

Schmidt, Annette (1985), Young People's Dyirbal: an Example of Language Death from Australia, Cambridge, Cambridge University Press.

Stipčević, Aleksandar (2011), Tradicijska kultura zadarskih Arbanasa, Zagreb, Ibis.

Tagliavini, Carlo (1937), L’Albanese di Dalmazia: contributi alla conoscenza del dialetto ghego di Borgo Erizzo presso Zara, Firenze, Leo S. Olschki Editore.

Tsunoda, Tasaku (2006), Language endangerment and language revitalization, Berlin, Mouton de Gruyter. 
Vuletić, Nikola (2014), «Les minorités linguistiques invisibles et/ou cachées de la Croatie : les communautés linguistiques arbënishtë, istro-roumaine et istriote », dans Ksenija Djordjević Léonard (dir.), Les minorités invisibles: diversité et complexité (ethno)sociolinguistiques, Paris, Michel Houdiard Éditeur, p. 182-192.

Wagner, Wolfgang, Robert Farr, Sandra Jovchelovitch, Fabio Lorenzi-Cioldi, Ivana Marková, Gerard Duveen et Diana Rosea (1999), «Theory and method of social representations », Asian journal of social psychology, vol. 2, n 1, p. 95-125. 